\title{
Molecular Origin for the Thermal Stability of S-88 Gum Produced by Pseudomonas ATCC 31554
}

\author{
Masakuni TAKO ${ }^{\dagger \dagger}$ and Hajime TAMAKI \\ Department of Bioscience and Biotechnology, University of the Ryukyus, \\ 1, Senbaru, Nishihara-cho, Okinawa 903-0213, Japan
}

(Received January 17, 2005; Accepted March 17, 2005; Published July 15, 2005)

\begin{abstract}
Non-Newtonian behavior and dynamic viscoelasticity of S-88 gum produced by Pseudomonas ATCC 31554 in aqueous solution were measured with a rheogoniometer. The S-88 gum showed shear-thinning behavior at a concentration of $0.1 \%$, but plastic behavior above $0.3 \%$. Gelation did not occur even at $1.0 \%$ and low temperature $\left(0^{\circ} \mathrm{C}\right)$. The viscosity decreased gradually with increasing temperature at concentrations below $0.3 \%$, but increased above $0.8 \%$, where a transition temperature was observed at $45^{\circ} \mathrm{C}$. The elastic modulus showed a constant value during increasing temperature at concentrations above $0.5 \%$. Gelation did not occur in the presence of $\mathrm{CaCl}_{2}(6.8 \mathrm{mM})$ even at a low $\left(0^{\circ} \mathrm{C}\right)$ temperature. A little decrease of the elastic modulus was observed in urea $(4.0 \mathrm{M}), 0.05 \mathrm{M} \mathrm{NaOH}$, and $85 \%$ DMSO solution and kept large values during increasing temperature. The thermal stability for viscosity and dynamic viscoelasticity of S-88 gum molecules might be attributed to intramolecular associations, possible mode of which was proposed. [DOI 10.1295/polymj.37.498]

KEY WORDS S-88 Gum / Dynamic Viscoelasticity / Molecular Origin / Thermal Stability / Hydrogen Bonding / van der Waals Interaction /
\end{abstract}

In the course of the rheological studies of polysaccharides, we have discussed the molecular origin for their rheological characteristics and proposed a gelation mechanism of $\kappa$-carrageenan, ${ }^{1,2}$ l-carrageenan, ${ }^{3}$ agarose(agar), ${ }^{4}$ gellan gum,,${ }^{5}$ amylose, ${ }^{6}$ curdlan,$^{7}$ and alginic acid ${ }^{8}$ at the molecular level in aqueous solutions. We also proposed synergistic gelation mechanism for a mixture solutions of xanthan and galactomannan (locust-bean gum, tara-bean gum and guar gum), ${ }^{9-14}$ and konjac glucomannan, ${ }^{14-16}$ where trisaccharide side-chains of the former moleculres take part in the interaction. Recently, we have proposed a gelatinization and retrogradation mechanisms of rice ${ }^{17,18}$ and potato starches. ${ }^{19}$ The short side-chains of rice and potato amylopectin molecules might take part in intermolecular hydrogen bonding with amylose molecules. Consequently, we have realized that there are some basic rules in the gel-formation processes of the polysaccharides in aqueous solutions. ${ }^{20}$ The gelation might occur in the formation of intra- and intermolecular associations, where hemiacetal oxygen, hydroxyl or methyl groups of the sugar residues of the polysaccharides contribute with hydrogen bonding or van der Waals interaction in aqueous solutions. The sulfuric accid and carboxyl groups of the sugar residues of some acidic polysaccharides, such as $\kappa$-carrageenan, $\iota$-carrageenan, gellan gum and alginic acid, might also participate inintra- or intermolecular associations through univalent or divalent cations with ionic bonding or electrostatic forces of attraction. On the basis of such gelation mechanisms, we have discussed the role of polysaccharides in gel-forming processes in water and demonstrated the principles of polysaccharide gels. ${ }^{20}$

S-88 Gum is a bacterial polysaccharide produced by Pseudomonas ATCC 31554, and is non-gel forming but gives a thermostable, highly viscous solution even at high temperatures $>100{ }^{\circ} \mathrm{C} .{ }^{21}$ The main chain of the polysaccharide consists of tetrasaccharide repeating units, 3$)-\beta$-D-Glcp-( $1 \rightarrow 4)-\beta$-D-GlcpA$(1 \rightarrow 4)-\beta$-D-Glcp-( $1 \rightarrow 4)-\alpha$-L-Rhap or $-\alpha$-L-Manp-( 1 , and every D-glucosyl residue next to the L-rhamnosyl or L-mannosyl resiue is substituted at O-3 by $\alpha$-Lrhamnosyl side-chain. ${ }^{22}$ The $\mathrm{S}-88$ gum also contains $O$-acetyl groups about $5 \%$, but the location of which have not been known yet. There is a remarkable similarity between S-88 and gellan gum, in that their repeating units all contain the same linear tetrasaccharide, unsubstituted for gellan gum, ${ }^{23,24}$ but substituted with mono-saccharide side chains for the S-88 gum.

As mentioned above, we have proposed a mode of intra- and intermolecular associations of gellan gum in aqueous solution, as illustrated in Scheme 1.5 The intramolecular hydrogen bonding takes place between $\mathrm{OH}-4$ group of the D-glucosyl residue and the adjacent hemiacetal oxygen atom of the L-rhamnosyl residue, and between OH-3 of the D-glucosyl residue and the adjacent hemiacetal oxygen atom of the

\footnotetext{
${ }^{\dagger}$ Paper presented at the 22nd International Carbohydrate Symposium, Glasgow, U.K., 23-27, July, 2004.

${ }^{\dagger \dagger}$ To whom correspondence should be addressed (E-mail: tako@eve.u-ryukyu.ac.jp).
} 


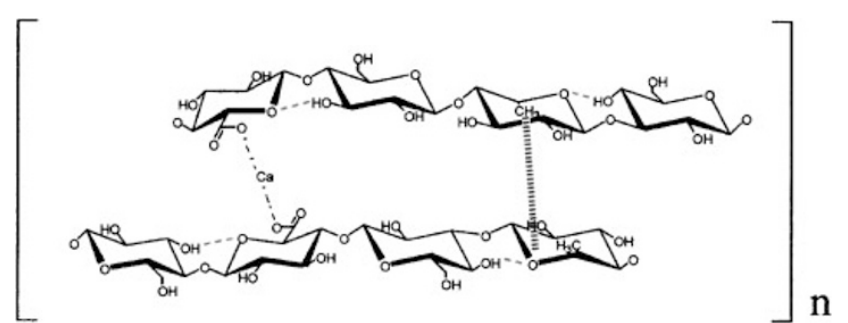

Scheme 1. Possible mode of intra- and intermolecular associations of gellan gum in aqueous solution. Association: - - - hydrogen bonding; ---- ionic bonding; / / / van der Waals interaction.

D-glucuronosyl residue, to make gellan gum molecule rigid. The intermolecular association takes place between the methyl group and the hemiacetal oxygen atom or the methyl group of the L-rhamnosyl residues on different molecules with van der Waals interaction by cage effect. ${ }^{3,4}$ In the presence of $\mathrm{CaCl}_{2}$, an intermolecular $\mathrm{Ca}^{2+}$ bridge, as in $\iota$-carrageenan ${ }^{3}$ and alginic acid, ${ }^{8}$ also takes place with ionic bonding between the carboxyl oxygen atom of the D-glucuronosyl residues on different molecules.

We have also proposed a model of intramolecular associations of welan ${ }^{25}$ and S- $657^{26}$ gum, the structure of which is similar to that of gellan, but substituted at $\mathrm{O}-3$ of every D-glucosyl residue next to the L-rhamnosyl residue by either $\alpha$-L-rhamnosyl or $\alpha$-L-mannosyl side chain in the ratio of $2: 1$ for the former, ${ }^{27}$ and substituted at O-3 of every D-glucosyl residue next to the L-rhamnosyl residue by $\alpha$-L-rhamnosyl disaccharide side chains for the latter ${ }^{28}$ in aqueous solution, as illustrated in Scheme 2 and Scheme 3. The intramolecular associations take place between the $\mathrm{OH}-4$ of the D-glucosyl residue and the adjacent hemiacetal oxygen atom of the L-rhamnosyl residue with hydrogen bonding as in gellan gum and between the methyl group of the L-rhamnosyl residue and the adjacent hemiacetal oxygen atom of the D-glucosyl residue with van der Waals interaction. The thermostability for viscosity and dynamic viscoelasticity of welan and S-657 gum has been attributed to the intramolecular associations, respectively. Though, the L-rhamnosyl residue adopted a ${ }^{4} \mathrm{C}_{1}$ pyranose-ring conformation

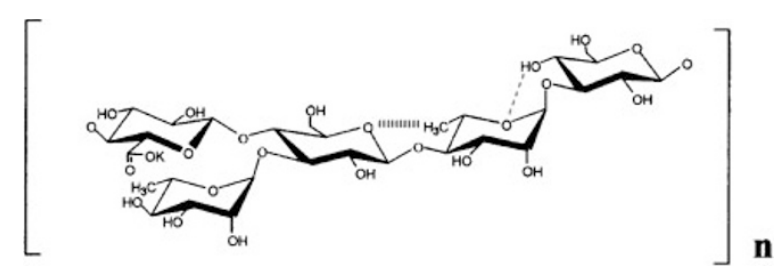

Scheme 2. Possible mode of intramolecular associations of welan gum in aqueous solution. Association: -----, hydrogen bonding; / / / van der Waals interaction.

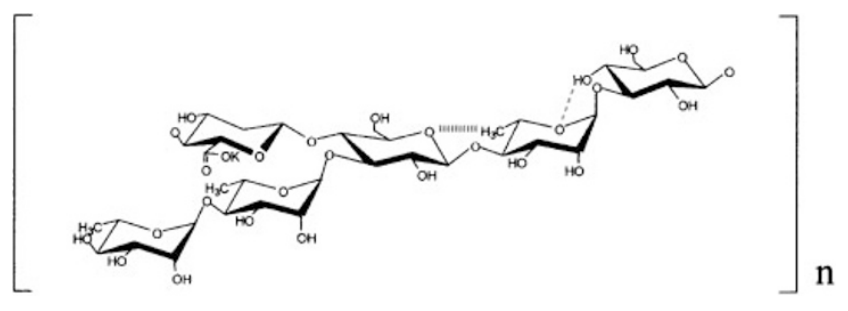

Scheme 3. Possible mode of intramolecular associations of S-657 gum in aqueous solution. Association: -----, hydrogen bonding; / / / van der Waals interaction.

in gellan gum molecules, ${ }^{5}$ in welan and S-657 gum molecules, it might existed in ${ }^{1} \mathrm{C}_{4}$ pyranose-ring conformation where the C-6 methyl group of the L-rhamnosyl residues on the main-chain adopted in equatorial orientation. Such conformational changes resulted in gelation for the gellan and non-gelling for welan ${ }^{25}$ and S-657 gums. ${ }^{26}$

On the other hand, the conformational change on the L-rhamnosyl residues has been taken place by alkali treatment to remove the acetyl group for native rhamsan molecules, ${ }^{29}$ which did not gel, but showed a thermostable, highly viscous and dynamiv viscoelastic solutions. ${ }^{30,31}$ The followed deacetylated rhamsan gum has formed a gelation where the methyl group at C-6 of the L-rhamnosyl residues adopted in axial orientation to associate with the hemiacetal oxygen atom or the methyl group of L-rhamnosyl residue on different molecules as in gellan (deacetylated) gum molecules. ${ }^{5}$ Such conformational change of L-rhamnosyl residues in rhamsan molecules has been confirmed by proton $\left({ }^{1} \mathrm{H}\right)$ NMR spectra. ${ }^{29}$

We report herein the flow behavior and dynamic viscoelasticity of S-88 gum, and its rheological properties are analysed with respect to its association characteristics, in comparison with those of deacetylated gellan, ${ }^{5}$ welan, ${ }^{25}$ and S- $657,{ }^{26}$ the structure of which are similar to that of the former.

\section{EXPERIMENTAL}

\section{Materials}

The S-88-gum was kindly supplied by CP-Kelco, California, U.S.A. and dissolved in hot water $\left(80^{\circ} \mathrm{C}\right)$ as a $0.1 \%$ solution. The solution was heated at $90^{\circ} \mathrm{C}$ for $20 \mathrm{~min}$, and then cooled at room temperature, centrifuged at 46,000 $g$ for $1 \mathrm{~h}$, and filtered through Celite 545 (which had been treated with boiling $3 \mathrm{M} \mathrm{HCl}$ for $30 \mathrm{~min}$ and washed with distilled water until $\mathrm{pH}$ 6.0). In the presence of $0.05 \% \mathrm{KCl}$, ethanol (2 vols.) was added to the filtrate and the precipitate was dried in vасио. Purified S-88 gum was redissolved in hot water and the solution was deionized by passage through a column of Amberlite IRA-120 $\left(\mathrm{H}^{+}\right)$, and neutralized 
with $0.05 \mathrm{M} \mathrm{KOH}$. The solution was filtered through Celite 545 again. Ethanol (2 vols.) was added to the filtrate in the presence of $0.05 \% \mathrm{KCl}$, and precipitate was dried in vacuo.

The gellan (deacetylated), welan and S-657 gum samples were also kindly supplied by CP-Kelco, California, U.S.A. and purified as previously described. ${ }^{5,25,26}$

Viscosity and Dynamic Viscoelasticity Measurements

To compare the rheological behaviors of S-88 gum with those of gellan, welan, S-657 and rhamsan gums, the viscosity and dynamic viscoelasticity were measured under the same conditions as those of previous studies. ${ }^{5,25,26,29-31}$

Viscosity at various shear rates $\left(1.19-95.05 \mathrm{~s}^{-1}\right)$ and dynamic viscoelasticity at an angular velocity (3.77 $\mathrm{rad} / \mathrm{s}$ ) were measured with a rheogoniometer equipped with a coaxial cylinder (18 $\mathrm{mm}$ diam.) and rotating outer cylinder (22 mm diam.), $60 \mathrm{~mm}$ long (IR-103, Iwamoto Seisakusho, Co., Ltd., Japan). Circulating oil from a Thermo-cool (LCH-130F, Toyo Co., Ltd., Japan) controlled the temperature of the sample over the temperature range from 0 to $85^{\circ} \mathrm{C}$, and raised it in steps at a rate of $1{ }^{\circ} \mathrm{C} / \mathrm{min}$. Shear rate $(\dot{\gamma})$, shear stress $(\tau)$ and apparent viscosity $(\eta)$ were calculated using the equation of Margules. ${ }^{32}$ Dynamic viscosity $\left(\eta^{\prime}\right)$ and elastic modulus $\left(\mathrm{G}^{\prime}\right)$ were calculated by a modification of Markovitz's equation. ${ }^{33}$ The mechanical loss tangent was calculated from the relationship $\tan \delta=\mathrm{G}^{\prime \prime} / \mathrm{G}^{\prime}$, where $\mathrm{G}^{\prime \prime}$ is the loss modulus $\left(\omega \eta^{\prime}\right)$ and $\omega$ is the angular velocity of the outer cylinder.

\section{RESULTS AND DISCUSSION}

\section{Flow Characteristic}

To compare the rheological behaviors of S-88 gum to those of gellan (deacetylated), welan and S-657 gum, the viscosity and dynamic viscoelasticity were measured under the same conditions as those of our previous studies. ${ }^{5,25,26}$ The flow curves, at $25^{\circ} \mathrm{C}$, of S-88 gum at various concentrations are shown in Figure 1. The flow curve at a concentration of $0.1 \%$ approximated to shear-thinning behavior, but to plastic behavior above $0.3 \%$, and the yield value was estimated to be 2.0, 4.0, 6.0 and $14.0 \mathrm{~Pa}$ at $0.3,0.5$, 0.8 , and $1.0 \%$, respectively. The yield value suggests that a secondary association is involved within or between S-88-gum molecules in aqueous solution. ${ }^{4,5,25,26,29-31}$ The flow curves of S-88 gum solutions shifted over high shear-stress in proportion to the concentrations up to $0.8 \%$. However, in $1.0 \%$ solution, it shifted very higher shear-stress than that in $0.8 \%$. This tendency essentially agreed with that of welan $^{25}$ and S-657 gum, ${ }^{26}$ but differed from that of

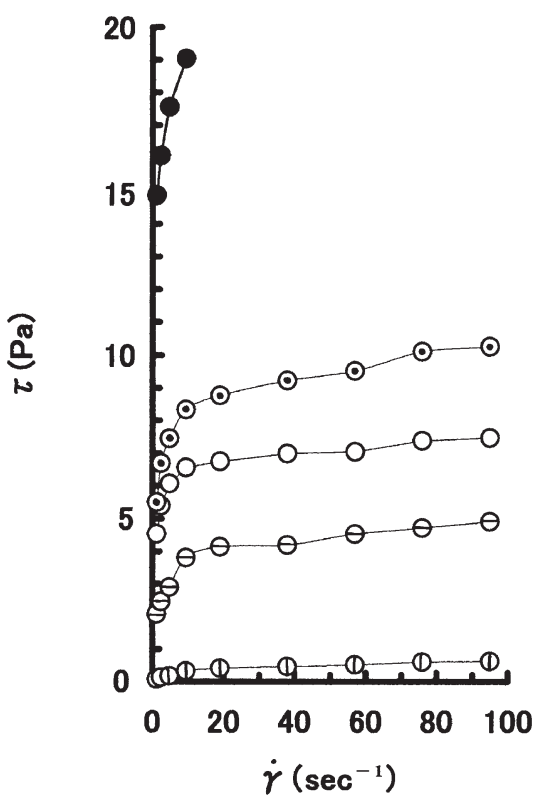

Figure 1. Flow curves of S-88 gum at various concentrations in aqueous solution at $25^{\circ} \mathrm{C}$. Concentration: $\Phi, 0.1 \%$; $\ominus, 0.3 \%$; $\bigcirc, 0.5 \% ; \odot, 0.8 \% ; \bullet, 1.0 \%$.

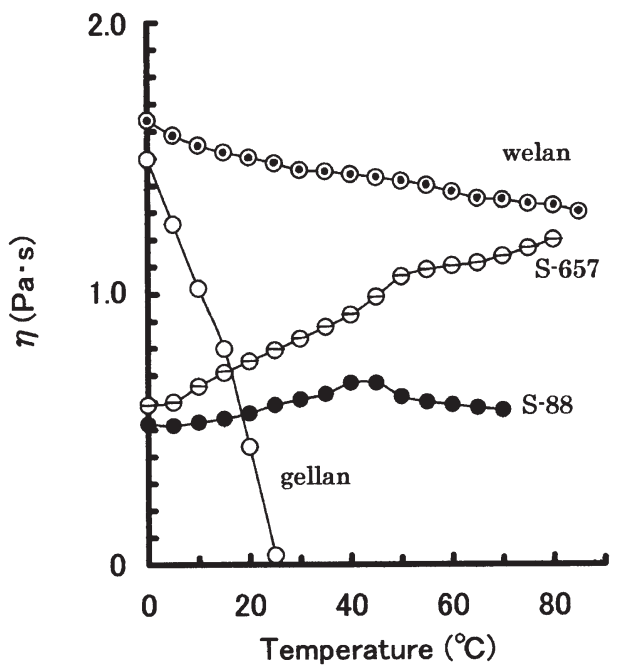

Figure 2. Effect of temperature on the viscosity of S-88, gellan, welan, and S-657 gums at $0.8 \%$ and $9.5 \mathrm{sec}^{-1}$. Symbols: -, S-88 gum; ○, gellan gum; ๑, welan gum; $\ominus$, S-657 gum.

gellan gum, ${ }^{5}$ where the flow curves shifted to a very low shear-stress below $0.9 \%$. This might be caused by the breakdown of an interrmolecular associattion of gellan gum, under a shear-rate of $9.50 \mathrm{~s}^{-1}$, since at $1.0 \%$ solution, it shifted over to very high shearstress and showed plastic behavior, the yield value of which was estimated to be $1.0 \mathrm{~Pa}^{5}$

The effect of temperature on the viscosity of S-88, gellan, welan and S-657 gums at a concentration of $0.8 \%$ was shown in Figure 2. Though viscosity of gellan gum showed large value at a low temperature $\left(0^{\circ} \mathrm{C}\right)$, it decreased rapidly with increasing tempera- 
ture. In contrast, the branched S-88 gum, welan and S-657 gum exhibited quite different viscosity properties. The viscosity of S- 88 gum stayed at low value at a low temperature $\left(0^{\circ} \mathrm{C}\right)$ and increased gradually with increasing temperature up to $45^{\circ} \mathrm{C}$, which was estimated to be a transition temperature, then it stayed at a cconstant value with further increasing temperature. The phenomenon is attributed to dissociation of a secondary association above the transition temperature. ${ }^{3,5,29,34-36}$ A similar transition temperature was also observed in S-657 gum at a temperature of $50{ }^{\circ} \mathrm{C} .{ }^{26}$ The viscosity of welan gum showed very large value at a low temperature and was steady during increase in temperature up to $80^{\circ} \mathrm{C}$.

The increase in viscosity was also observed in a solution of native xanthan, ${ }^{34}$ deacetylated, ${ }^{35}$ depyruvated ${ }^{36}$ and deacylated. ${ }^{37}$ This was attributed not only to its formation of intramolecular associations (to which the methyl group of the acetyl residues and the OH-3 of the D-glucosyl residues contributed), but also to an increase of kinetic energy of the trisaccharide side-chains of xanthan. However, the viscosity of deacylated xanthan showed a maximum value at 25,35 , and $40^{\circ} \mathrm{C}$ at concentrations of $0.5,0.8$, and $1.0 \%$, respectively, then it decreased rapidly. ${ }^{37}$ The decrease in the viscosity was attributed to free from acetyl and pyruvate groups. Accordingly, the increase in viscosity with increasing temperature of the S-88 gum may be due to an formation of a secondary association and to an increase in kinetic energy of the sidechains. Thus, rapid increase in the viscosity for S-657 gum $^{24}$ with increasing temperature may be due to increase in kinetic energy of disaccharide side-chains.

As shown in Figure 3, the viscosity of S-88 gum

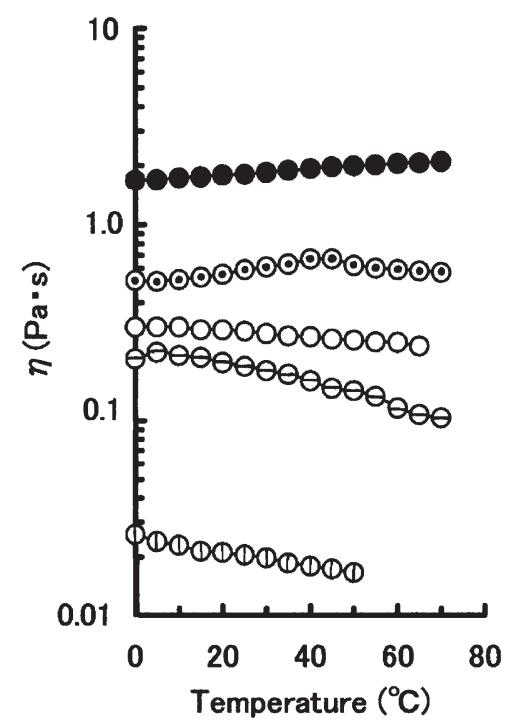

Figure 3. Effect of temperature on the viscosity of S-88 gum at various concentrations. Concentration: $\odot, 0.1 \%$; $\ominus, 0.3 \%$; ○, $0.5 \% ; \odot, 0.8 \% ; \bullet, 1.0 \%$. decreased gradually at concentrations below $0.3 \%$, but it stayed at a constant value in a solution of $0.5 \%$ with increasing temperature. The viscosity for $0.8 \%$ solution increased gradually with increasing temperature up to $45^{\circ} \mathrm{C}$, then it stayed at a constant value with further increase in temperature, as mentioned above (Figure 2). In a solution of $1.0 \%$, the viscosity increased gradually with increasing temperature up to $70^{\circ} \mathrm{C}$. These suggest that $\mathrm{S}-88$ gum molecules may involve an intramolecular association to which the methyl group of the L-rhamnosyl residue contribute, since thermostable phenomenon might be attributed to involving the methyl group in xanthan, ${ }^{14,35-37}$ welan, ${ }^{25}$ rhamsan, ${ }^{30,31}$ and S-657. ${ }^{27}$

\section{Dynamic Viscoelasticity}

As reported previously, the elastic modulus of gellan gum solution was very large at a concentration of $0.8 \%$ at low temperature $\left(0^{\circ} \mathrm{C}\right)$, but decreased rapidly with increasing temperature. ${ }^{5}$ The elastic modulus, however, of welan, ${ }^{25}$ rhamsan, ${ }^{30,31}$ and S-657 gums ${ }^{27}$ showed large value during increase in temperature up to $80^{\circ} \mathrm{C}$. The elastic modulus of S-88 gum showed low values even at low temperature $\left(0^{\circ} \mathrm{C}\right)$ and decreased gradually with increasing temperature at a concentration of $0.1 \%$, as shown in Figure 4. The elastic modulus increased with increase in concentration and stayed large value during increase in temperature up to $0.8 \%$ solution. However, almost the same elastic modulus was observed in $1.0 \%$ solution as compared in $0.8 \%$ solution and kept constant values with increasing temperature up to $70^{\circ} \mathrm{C}$, which was estimated to be a transition temperature, then it decreased a little with further increase in temperature.

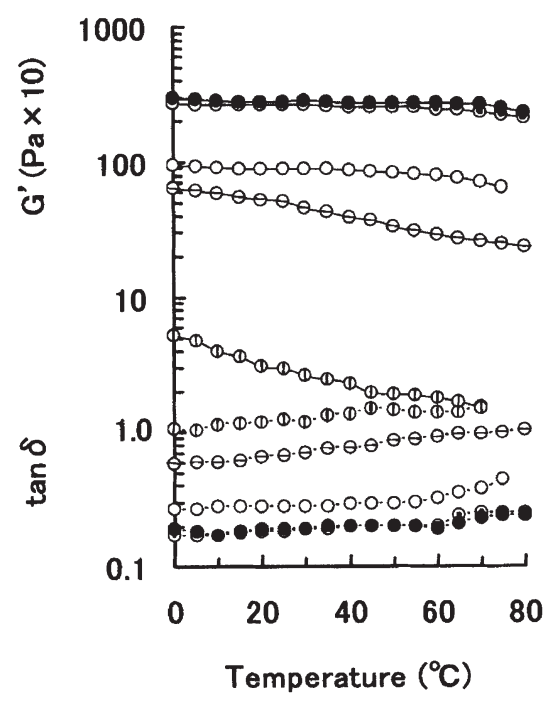

Figure 4. Effect of temperature on elastic modulus of S-88 gum at various concentrations. Concentration: $\Phi, 0.1 \% ; \ominus, 0.3 \%$; $\bigcirc, 0.5 \% ; \odot, 0.8 \% ; \bullet, 1.0 \%$. 
Though $\tan \delta$ decreased from 1.05 to 0.20 with increase in concentration from 0.1 to $0.8 \%$, it increased a little to 0.22 with further increase in concentration to $1.0 \%$ at low temperature $\left(0^{\circ} \mathrm{C}\right)$. The values stayed at a constant with increasing temperature up to $70^{\circ} \mathrm{C}$ which was estimated to be a transition temperature, then it increased a little with further increase in temperature. Such phenomenon, staying large elastic modulus at high temperature range, are in agreement with those of welan, ${ }^{25}$ rhamsan, ${ }^{30,31}$ and S-657 gums. ${ }^{27}$ The transition temperature, $70{ }^{\circ} \mathrm{C}$, indicates that a secondary association of S-88 gum molecules dissociate above the temperature. The almost identical elastic moduli in both 0.8 and $1.0 \%$ solutions during increasing temperature suggest that an intramolecular association involve in S-88 gum molecule in aqueous solution. Because a little increase of the elastic modulus was observed when the concentration of rhamsan gum increased from 0.8 to $1.0 \%$, as reported previously. ${ }^{30,31}$ On the contrary, decrease of the elastic modulus was observed in S-657 gum when concentration was increased in $1.0 \% .^{27}$

A very large elastic modulus was observed on addition of $\mathrm{CaCl}_{2}(6.8 \mathrm{mM})$ to a $0.2 \%$ solution of gellan gum, which increased further with increasing temperature up to $80^{\circ} \mathrm{C}$, and then decreased rapidly which was estimated to be a transition temperature, as shown in Figure 5. This behavior indicates that gellan gum tightly binds $\mathrm{Ca}^{2+}$, with the carboxyl groups of the D-glucuronosyl residues contributing to cation-bridges between the different molecules with ionic bonding, ${ }^{5}$ because very small elastic modulus was observed in solution of polysaccharide alone. On the contrary,

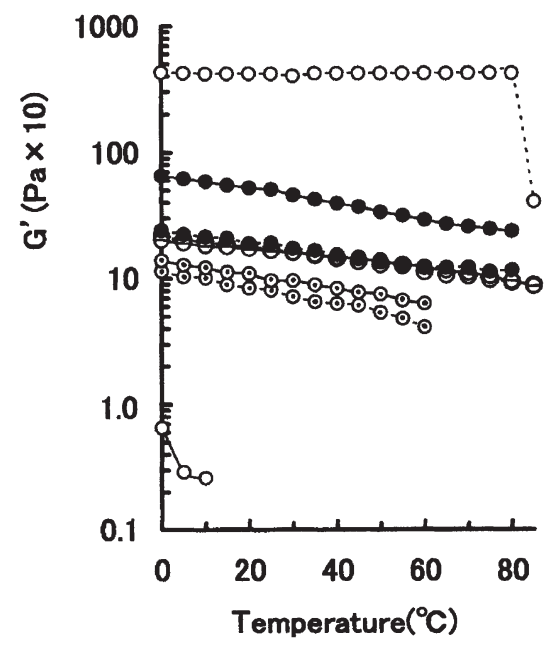

Figure 5. Effect of temperature on elastic modulus of S-88 gum $(0.2 \%)$ with addition of $\mathrm{CaCl}_{2}(6.8 \mathrm{mM})$. The solid lines refer to aqueous solution and dotted lines to addition of $\mathrm{CaCl}_{2}$. Symbols: ๑, S-88 gum; ○, gellan gum; ๑, welan gum; $\ominus$, S-657 gum. the elastic modulus of S-88 gum solution (0.2\%) decreased with addition of $\mathrm{CaCl}_{2}$, indicating that the side-chains of S-88 gum prevent the formation of $\mathrm{Ca}^{2+}$ bridges by the L-rhamnosyl side-chains. Such decrease of the elastic modulus was also observed in welan gum solution ${ }^{25}$ with addition of $\mathrm{CaCl}_{2}$. On the other hand, the elastic modulus of S-657 gum showed almost identical values on addition of $\mathrm{CaCl}_{2}$ with increase in temperature as solution of the polysaccharide alone. ${ }^{25}$ These also indicate that the welan and S-657 gum side-chains prevent the formation of $\mathrm{Ca}^{2+}$ bridges on the carboxyl groups of the D-glucuronosyl residues between different molecules.

Though very large increase of the elastic modulus was observed upon addition of urea $(4.0 \mathrm{M})$ to $0.8 \%$ gellan gum solution and gel formation was observed at low temperature $\left(0^{\circ} \mathrm{C}\right)$, it decreased rapidly with increasing temperature as solution of polysaccharide alone. For S-88 gum, however, a small decrease of the elasttic modulus was observed with addition of urea $(4.0 \mathrm{M})$ and remained large value with increasing temperature, as in case of the polysaccharide alone, as shown in Figure 6. The result suggests that a hydrophobic interaction contributing methyl groups of the L-rhamnosyl residues may participate in the interaction, because urea is one of hydrogen bonding breaker.

The elastic modulus decreased when S-88 gum was dissolved in $0.05 \mathrm{M} \mathrm{NaOH}$ solution even at low $\left(0^{\circ} \mathrm{C}\right)$ temperature as compared with its aqueous solution and decreased gradually with increasing temperature, as shown in Figure 7. Though a little decrease of dynamic viscosity was observed, $\tan \delta$ showed larger than that in aqueous solution and increased gradually

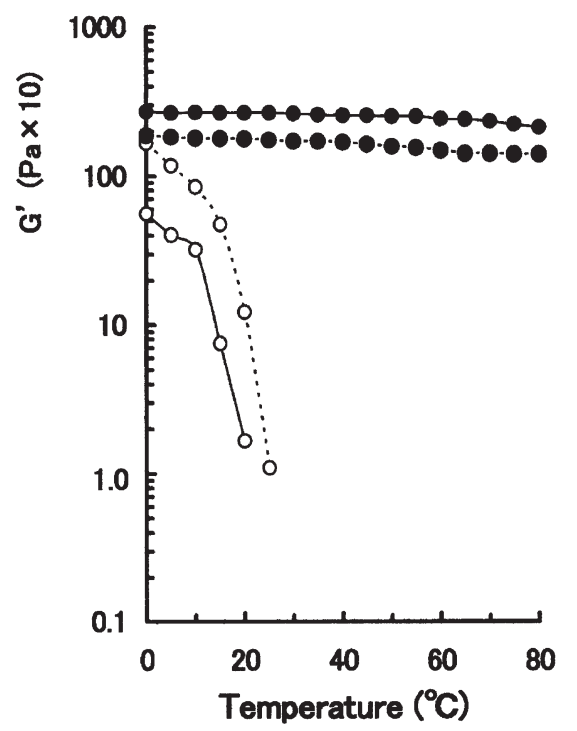

Figure 6. Effect of temperature on elastic modulus of S-88 gum at a concentration of $0.8 \%$ with addition of urea $(4.0 \mathrm{M})$. The solid lines refer to aqueous solution and dotted lines to addition of urea. Symbols: $\bullet$ S-88 gum; $\bigcirc$, gellan gum. 


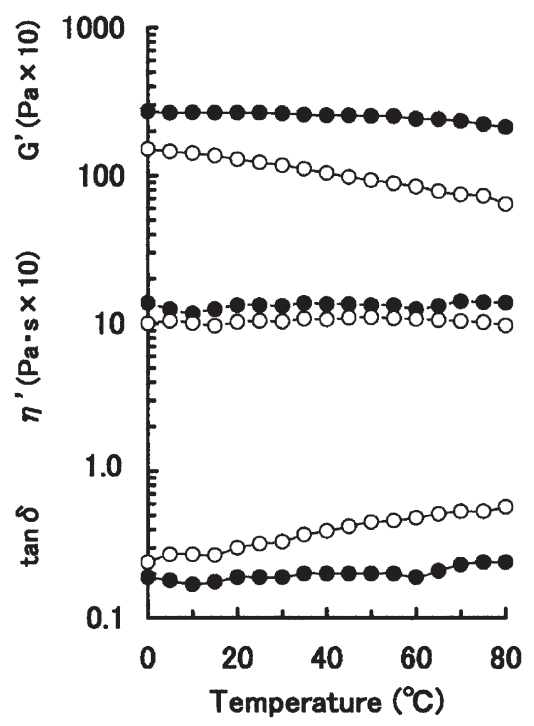

Figure 7. Effect of temperature on dynamic viscoelasticity of S-88 gum at a concentration of $0.8 \%$ in alkali $(0.05 \mathrm{M} \mathrm{NaOH})$ solution. Symbols: $\bullet$, aqueous solution; $\bigcirc$, in $\mathrm{NaOH}$ solution.

with increasing temperature. The result indicates that the secondary association dissociated in part in $0.05 \mathrm{M} \mathrm{NaOH}$.

Almost identical elastic modulus was observed at low $\left(0{ }^{\circ} \mathrm{C}\right)$ temperature when S- 88 gum was dissolved in $85 \%$ dimethyl sulfoxide (DMSO), but decreased gradually with increasing temperature, as shown in Figure 8. Identical dynamic viscosity and $\tan \delta$ were also observed in $85 \%$ DMSO solution as compared with that in aqueous solution during increasing temperature. The result suggests that a hydrophobic interaction contributing the methyl group of the L-rhamnosyl residues may participate in the interaction, because DMSO is a hydrogen bonding breaker.

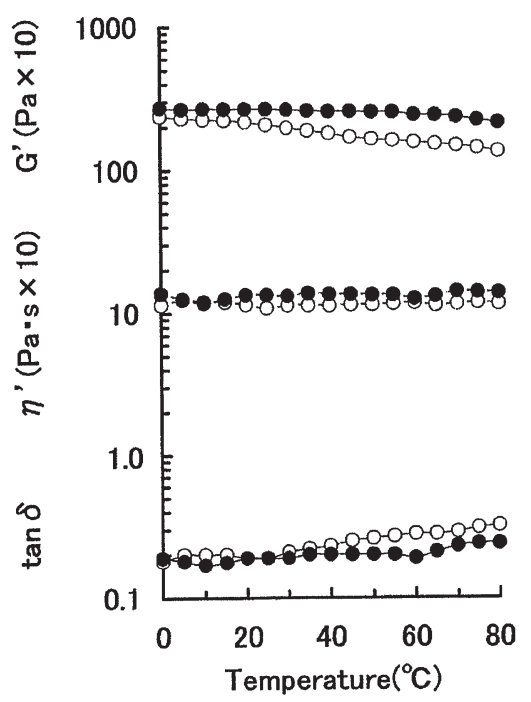

Figure 8. Effect of temperature on dynamic viscoelasticity of S-88 gum at a concentration of $0.8 \%$ in $85 \%$ DMSO. Symbols: •, aqueous solution; $\bigcirc, 85 \%$ DMSO.

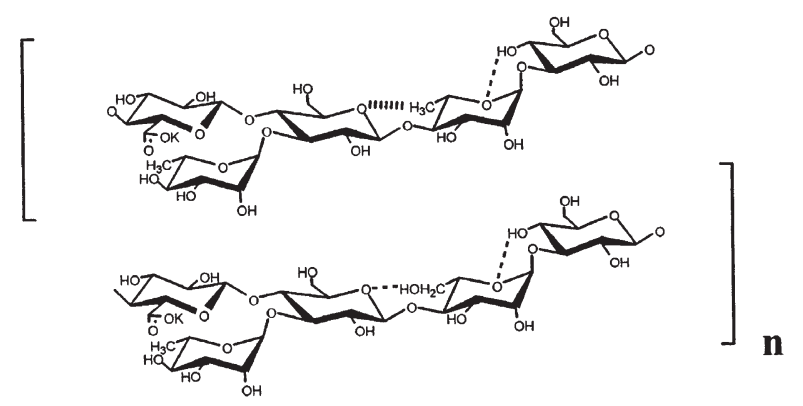

Scheme 4. Possible mode of intrarmolecular associations of S-88 gum in aqueous solution. Association: - - - - hydrogen bonding; / / / van der Waals interaction.

\section{CONCLUSIONS}

Though the primary structure of S-88 gum is similar to that of gellan (deacetylated), welan, rhamsan, and S-657 gums, its rheological characteristics differed from those of the gellan, but essentially agreed with those of welan, rhamsan, and S-657 gums. Accordingly, the S-88 gum molecules may involve intramolecular associations, as do welan, rhamsan, and S-657 molecules. As shown in Scheme 4, intramolecular associations may take place between $\mathrm{OH}-4$ of the D-glucosyl residue and adjacent hemiacetal oxygen atom of the L-rhamnosyl or L-mannosyl residue with hydrogen bonding, and between the methyl or hydroxylmethyl group of the L-rhamnosyl or L-mannosyl residue and adjacent hemiacetal oxygen atom of the D-glucosyl residue with van der Waals interaction or hydrogen bonding. The intramolecular associations play a dominant role in thermostable characteristic in S-88 gum where the L-rhamnosyl residue on the main chain contribute. The L-rhamnosyl residue may adopt ${ }^{1} \mathrm{C}_{4}$ pyranose-ring conformation in part in aqueous solution, as in welan, ${ }^{25}$ rhamsan ${ }^{30,31}$ and S657 gum. ${ }^{27}$

Especially, the S-88 and welan gum is substituted the same L-rhamnosyl mono-saccharide side-chain at the same position of the main-chain (at C-3 of Dglucosyl residue). ${ }^{20,37}$ However, the structure of the both polysaccharides differes only $1 / 2$ of L-rhamnosyl residues are replaced to L-mannosyl residues on tetrasaccharide repeating units of the main-chain for S- 88 gum where the both sugar residues, L-rhamnosyl and L-mannosyl residues, differed only at C-6; methyl group for the former and hydroxyl methyl group for the latter. ${ }^{2}$ Such different substitution may contribute to their rheological characteristics: Though the shear-stress increased with increasing shear-rate up to $57.0 \mathrm{~s}^{-1}$ in welan gum solution, in the case of S-88 gum, it increased only up to $9.5 \mathrm{~s}^{-1}$, then stayed at a constant shear-stress with further increase in shearate (Figure 1), indicating that S-88 gum molecules 
were liable to orient to shearing force as in xanthan molecules. ${ }^{34-37}$ The viscosity of welan gum $(0.8 \%)$ showed very large at low temperature, however, that of S-88 gum $(0.8 \%)$ stayed at low values, probably because of orientation of the molecules along shearing force, and increased gradually with increasing temperature up to $45^{\circ} \mathrm{C}$, which was estimated to be a transition temperature, then it stayed at a constant value. The transition temprature indicates that a part of intramolecular associations dissociate above the transition temperature. Though almost identical viscosity was observed in rhamsan gum solution (Figure 3) compared with that of S-88 gum at low temperature $\left(0{ }^{\circ} \mathrm{C}\right)$, it increased rapidly with increasing temperature up to $50^{\circ} \mathrm{C}$, which was estimated to be a transition temperature, then increased a little. The transition temperature may be caused by dissociation of intramolecular hydrogen bonding contributing disaccharide side-chain with glycan backbone of rhamsan gum molecule. ${ }^{30,31} \mathrm{~A}$ transition temperature was also observed for elastic modulus and $\tan \delta$ in S-88 gum solution, but not in welan gum (Figure 5). Thus, the transition temperature in S-88 gum solution may be caused by a dissociation of the intramolecular hydrogen bonding where C-6 of the L-mannosyl residue with the adjacent hemiacetal oxygen atom of the Dglucosyl residue contributed. The more large elastic modulus in S-88 gum solution (Figure 6) than that in welan gum may be due to formation of hydrogen bonding, where L-mannosyl residue contribute, the strength of which is larger than that of van der Waals interaction where the methyl group of the L-rhamnosyl residues contributed, but the hydrogen bonding may dissociates above the transition temperature at $45^{\circ} \mathrm{C}$ under shearing force and at $70^{\circ} \mathrm{C}$ under frequency. The dissociation of hydrogen bonding occurred in agarose $e^{4}$ and potato amylose ${ }^{6}$ molecules at a temperature of 60 and $80^{\circ} \mathrm{C}$ under frequency, respectively in aqueous solution. Under shearing force, however, the hydrogen bonding dissociated at 35 and $90^{\circ} \mathrm{C}$ in the potato amylose molecules. The result indicated that a two-steps mechanism existed for a conformational transition in potato amylose molecules under shearing force. ${ }^{6}$

Consequently, Scheme 4 provides an explanation not only for the rheological characteristics of the S-88 gum, but also for those of gellan, ${ }^{5}$ welan, ${ }^{25}$ rhamsan, ${ }^{30,31}$ and S-657 $7^{27}$ gums in aqueous solutions. The model corresponds to a single-stranded helix.

Acknowledgment. The authors thanks Dr. J. Baird and Dr. T. A. Talashek, CP-Kelco, California, U.S.A., for providing the S-88, gellan, welan, rhamsan, and S-657 gums.

\section{REFERENCES}

1. M. Tako and S. Nakamura, Carbohydr. Res., 155, 200 (1986).

2. M. Tako and S. Nakamura, Agric. Biol. Chem., 50, 2817 (1986).

3. M. Tako, S. Nakamura, and Y. Kohda, Carbohydr. Res., 161, 247 (1987).

4. M. Tako and S. Nakamura, Carbohydr. Res., 180, 277 (1988).

5. M. Tako, A. Sakae, and S. Nakamura, Agric. Biol. Chem., 53, 771 (1989).

6. M. Tako and S. Hizukuri, J. Carbohydr. Chem., 14, 613 (1995).

7. M. Tako and I. Hanashiro, Polym. Gels Networks, 5, 241 (1997).

8. M. Tako and Y. Kohda, J. Appl. Glycosci., 44, 153 (1997).

9. M. Tako, A. Asato, and S. Nakamura, Agric. Biol. Chem., 48, 2995 (1984).

10. M. Tako and S. Nakamura, Carbohydr. Res., 138, 207 (1985).

11. M. Tako and S. Nakamura, FEBS Lett., 204, 33 (1986).

12. M. Tako, J. Carbohydr. Chem., 10, 619 (1991).

13. M. Tako, Carbohydr. Polym., 15, 227 (1991).

14. M. Tako, ACS Symp. Ser., 489, 268 (1992).

15. M. Tako, Colloids Surf., B, 1, 125 (1992).

16. M. Tako, Biosci., Biotechnol., Biochem., 56, 1186 (1992).

17. M. Tako and S. Hizukuri, J. Carbohydr. Chem., 18, 573 (1999).

18. M. Tako and S. Hizukuri, Cereal Chem., 77, 473 (2000).

19. M. Tako and S. Hizukuri, Carbohydr. Polym., 48, 397 (2003).

20. M. Tako, J. Appl. Glycosci., 47, 49 (2000).

21. R. Moorhouse, in "Industrial Polysaccharides," M. Yalpani, Ed., Elsevier, Amsterdam, 1987, p 187.

22. P.-E. Jansson, N. S. Kumar, and B. Lindberg, Carbohydr. Res., 156, 165 (1986).

23. M. A. O'Neill, R. P. Selvendran, and V. J. Morris, Carbohydr. Res., 124, 123 (1983).

24. P.-E. Jansson, B. Lindberg, and P. A. Sandford, Carbohydr. Res., 124, 135 (1983).

25. M. Tako and M. Kiriaki, Agric. Biol. Chem., 54, 3097 (1990).

26. M. Tako, Polym. Gels Networks, 2, 358 (1994).

27. P.-E. Jansson, B. Lindberg, G. Wildmalm, and P. A. Sandford, Carbohydr. Res., 139, 213 (1985).

28. T. A. Chowdhury, B. Lindberg, U. Lindberg, and J. Baird, Carbohydr. Res., 164, 117 (1987).

29. M. Tako, S. Tohma, T. Taira, and M. Ishihara, Carbohydr. Polym., 54, 279 (2003).

30. M. Tako, Biosci., Biotechnol., Biochem., 57, 1182 (1992).

31. M. Tako, in "Carbohydrates and Carbohydrate Polymers," M. Yalpani and M. Yalpani, Ed., ATL Press, Shrewsbury, MA, 1993, p 206.

32. J. Harris, in "Rheology and non-Newtonian flow," Longman, New York, N.Y., 1977, p 28.

33. H. Markovitz, J. Appl. Phys., 23, 1070 (1952).

34. M. Tako, T. Nagahama, and D. Nomura, Nippon Nogei 
Molecular Origin for the Thermal Stability of S-88 Gum Produced by Pseudomonas ATCC 31554

Kagaku Kaishi, 51, 513 (1977).

35. M. Tako and S. Nakamura, Agric. Biol. Chem., 48, 2987 (1984).

36. M. Tako and S. Nakamura, Agric. Biol. Chem., 52, 1585
(1988).

37. M. Tako and S. Nakamura, Agric. Biol. Chem., 53, 1941 (1989). 